\title{
Macroinvertebrates communities of a coastal lagoon in southern Benin, West Africa
}

\author{
Delphine ADANDEDJAN ${ }^{1 *}$, Philippe LALEYE $^{1}$ et Germain GOURENE ${ }^{2}$ \\ ${ }^{1}$ Laboratoire d'Hydrobiologie et d'Aquaculture, FSA-UAC. Faculté des Sciences Agronomiques, \\ Université d'Abomey-Calavi, 01 BP 526, Cotonou, Benin. \\ ${ }^{2}$ Laboratoire d'Environnement et de Biologie Appliquée U.F.R.-S.G.E Université d'Abobo-Adjamè, \\ 02 BP 801 Abidjan 02, Côte d'Ivoire. \\ ${ }^{*}$ Corresponding author, E-mail: adandedjandelph@yahoo.fr
}

\begin{abstract}
The macroinvertebrate, assemblage investigated were collected in eight seasonal samplings from July 2007 through June 2009. A total of 4,179 individual macroinvertebrates were gathered, comprising 182 taxa belonging to 25 orders and 114 families. The most predominant groups were Molluscs and Crustacean which make up $71.11 \%$ of the taxonomic richness observed. Annelids and Insects were the second most predominant. This restricted biodiversity is based on a limited number of species such as Tympanotonus fuscatus radula, Pachymelania aurita, Neritina glabrata, Corbula sp., Clibanarius spp., Grandidierella africana, Nereis sp. and Chironomus sp.. Site typology based on environmental parameters reveals five assemblages in which no significant difference exists. The community is controlled by a salinity gradient but is also probably affected by hydrologic factors and human economic activities.
\end{abstract}

(C) 2012 International Formulae Group. All rights reserved.

Keywords: Macroinvertebrates, communities, distribution, abundance, diversity, lagoon.

\section{INTRODUCTION}

Diversity in macroinvertebrates populations reveals remarkable temporal and spatial variations in coastal wetlands (Akin et al., 2003; Alfaro, 2006). Monitoring changes in water quality in aquatic ecosystems can be performed through an investigation of the macroinvertebrates living therein. Benthic macroinvertebrates characteristically respond to anthropic disturbances in an integrated and continuous manner thanks to their taxonomic diversity, their sedentary behaviour, and relatively long life cycles (Chaouti and Bayed, 2005; Edia et al., 2007). As a result, they have been widely used to assess water quality and the ecological state of aquatic ecosystems (Park et al., 2003; Edia et al., 2007; Fishar and Williams, 2008). Using macroinvertebrates as indicator, water quality has been found to fit on a scale from "clean" to "severely polluted" (Bazaîri et al., 2003; Alfaro, 2006). However, further investigation is needed to understand some of the features which characteristize the ecology of the macroinvertebrates taxa encountered in those environments.

In Africa, particularly in the Republic of Benin, there has been a gradual build-up of literature on macroinvertebrates and their use as bioindicators. Few inventories of benthic organisms exist in Benin and those inventories 
do not go in-depth in their characterization of macroinvertebrates neither do they extend much on their use as water quality indicators. However, an important step in achieving the sustainable management of aquatic ecosystems is a solid understanding of community abundance patterns. This is because community composition depends on habitat stability which makes possible the growth of resident populations. Next, the structure of aquatic communities is also function of various environmental factors which influence the habitats in both space and time. As an example, the distribution of macrobenthic communities is sometimes correlated with the type of sediment, which in turn is related to a wider set of environmental parameters such as current velocity and organic content of sediments (Snelgrove and Butman 1994; Gray et al., 2002). These complex relationships between community variations and environmental disturbances can be studied through community composition (structure or functional perspective) (Park et al., 2003) to establish the patterns of species biodiversity.

The present study aims at analysing the community patterns of macroinvertebrates collected in a coastal lagoon in southern Benin and elucidating the association of community clusters with the taxa-abundance patterns and its diversity.

\section{MATERIALS AND METHODS}

\section{Study area}

The study was undertaken in the southern part of Benin in the Coastal Lagoon situated between $2^{\circ} 16^{\prime} \mathrm{W}$ and $6^{\circ} 20^{\prime} \mathrm{N}$. The lagoon's surface area varies from 40 to $52 \mathrm{~km}^{2}$ in low and high waters, respectively. It is about $60 \mathrm{~km}$ long and 0,2-1 km wide. It is located along the Atlantic Ocean between Grand-Popo and Togbin and is bordered to the north by the towns of Grand-Popo and Ouidah, and to the east by a swampy area of Paspalum vaginatum. To the west, it joins the Gbagan River at Anecho City before reaching the sea via the Boca del Rio delta (Figure 1). Ecologically, the lagoon is a sub-unit of the RAMSAR site 1017. Its water has two origins: a marine origin through tidal actions and a freshwater origin via the Mono-Sazué and Couffo-Ahémé-Aho Rivers. Since the completion of the Nangbéto hydroelectricity dam on the Mono River, the hydrologic regime of the lagoon has changed due to water releases from the dam which considerably reduce its salinity.

The climate is equatorial with two rainy seasons and two dry seasons. During the rainy season, the lagoon is covered by floating Eichhornia crassipes, Pistia stratiotes, aquatic and palustrine weeds (Echinochloa spp., Panicum sp., etc.) which produce a patchy distribution of decaying litter. Human activities in the lagoon include fishing, ecotourism, salt production, and oyster farming. Much of the lagoon is surrounded by mangroves, Elaeis guineensis and coconut trees. Wastes of all kinds (solid household wastes, wastewater, etc.) are commonly discarded into the lagoon.

\section{Sampling}

Macroinvertebrate and sediment samples have been collected seasonally, in 8 phases from July 2007 to June 2009 at 18 sites (Figure 1). Corresponding environmental parameters were recorded. Sites were located by GPS on each sampling occasion.
Abiotic variables
Salinity, water depth, water transparency and dissolved oxygen were measured in situ at each sampling site. The salinity, conductivity and Total Dissolved Solids (TDS) were determined using a multiprocessor conductimeter; the dissolved oxygen concentration (DO) was measured with an oxymeter probe, while the water transparency and water depth were measured using a Secchi disk. The bottom sediment texture was determined in the laboratory by passing each sample through a series of sieves 


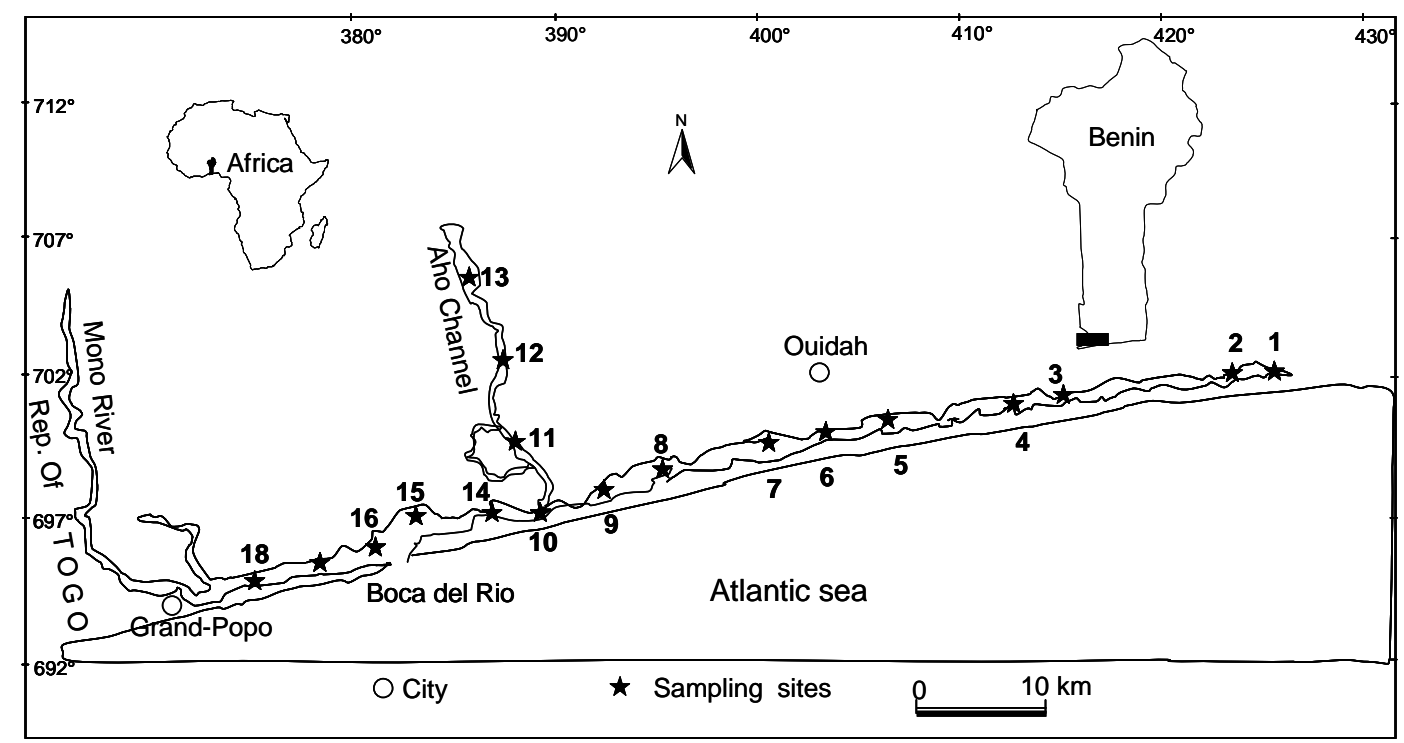

Figure 1: Locations of 18 sample sites in the Coastal Lagoon. Numbers represent sample site.

$1=$ Togbin 1, $2=$ Togbin 2, 3 = Avlékété $1,4=$ Avlékété $2,5=$ Djègbadji $1,6=$ Djégbadji 2, $7=$ Djégbadji $3,8=$ Azizakouè $1,9=$ Azizakouè $2,10=$ Djondji, $11=$ Aho $1,12=$ Aho $2,13=$ Aho $3,14=$ Hokouè, $15=$ Docloboué, $16=$ Alongo, 17 = Avlo, 18 = Agonnékanmè.

of different mesh sizes; then the percentage contribution of sand and fine particles (silt and clay) in each sediment sample was determined.

\section{Macroinvertebrates}

At each sampling site, 8 samples were collected with an Ekman grab sampler of 225 $\mathrm{cm}^{2}$ at a depth of approximately $10 \mathrm{~cm}$. The grabs were taken from random spots at each site. Each sample was sieved through two sieves of $500 \mu \mathrm{m}$ and $1 \mathrm{~mm}$ mesh size in the lagoon water and the collected organisms were cleaned under lagoon water and preserved separately in labelled bottles containing 4\% formaldehyde solution. Those meshes sizes were used because macroinvertebrates are almost always greater than $1 \mathrm{~mm}$ while Gastropods are generally of a size superior to $1 \mathrm{~mm}$ while Annelids, insect larvae and nymphs can be less than $0.5 \mathrm{~mm}$. So, using a mesh less than the size of the smaller macroinvertebrate would ensure that all the invertebrates are retrieved. Once in the laboratory, the invertebrate specimens from each site were sorted and identified. The specimens were then counted under microscope and finally preserved in $70 \%$ alcohol.

\section{Data analyses}

The mean values of abiotic parameters were calculated. The spatial variations of the benthic assemblages were determined using the artificial non-supervised neuron networks, the "Self Organizing Maps (SOM)" or Kohonen maps (Kohonen, 1982). The SOM architecture consisted of two layers of neurons: the input layer made of 70 neurons connected to each vector (line of the matrix) and the output layer made of 36 neurons. We have chosen 36 neurons because the configuration obtained presented minimum values for both quantification and topographic errors, which are used to appreciate the classification quality (Park et al., 2003). The SOM algorithm calculates the connection intensities between input and output layers by using an unsupervised competitive learning procedure (Kohonen, 1995). This classifies samples in each node according to their similarity in the environment. The analysis was carried out using the SOM toolbox for Matlab. The relevant groups or sample 
clusters which characterize the sampling sites assemblages were determined by performing a hierarchical classification analysis (Ward's linkage and the Euclidean distance method). The number of clusters was defined by applying the Bouldin-Davies Index (DBI), in which the minimum values indicate some low variance within groups and high variance between groups (Vesanto et al., 1999).

Number of taxa (richness), abundance, Shannon diversity index (H') and Pielou evenness (E) were calculated as descriptive measures of the benthic community of each group of sites as defined by the typology. Differences in each abiotic or biotic parameter calculated between clusters were tested by applying the non-parametric test of KruskalWallis. The spatial effect was tested by the Mann-Whitney test. For these different tests, we used STATISTICA 4.5.

\section{RESULTS}

\section{Environmental factors}

Table 1 shows the six mean values of the environmental parameters investigated in this study. For water salinity, transparency, and dissolved oxygen mean values, significant differences were noted (ANOVA, $\mathrm{p}<0.01$ ). As expected, water salinity increased seaward along the lagoon, from $0 \mathrm{mg} / \mathrm{L}$ (in the upper side) to $26.5 \mathrm{mg} / \mathrm{L}$ (in the channel Aho). Along the lagoon, there was no significant variation of the depth ( $\mathrm{p}>0.05)$. Sedimentological characteristics showed that the bottom of the lagoon was sandy with some muddy sites.

\section{Clusters identification}

Figures 2 and 3 showed the SOM map and the hierarchical classification of the samples on the basis of environmental variables. Five groups of samples were defined from the typological analysis. Group I (ot1) comprised 12 samples from the lower part of the lagoon characterized by high depth values with the substrate dominated by fine sediment particles. Group II (gal) comprised 17 samples from the upstream part of the lagoon. This group was distinguished by a sandy substrate and high DO values. Group III (od2) comprised 15 samples which were essentially from the central part of the lagoon. It is characterized by high salinity values, water transparency with a sandy-muddy substrate. Group IV (oa1), comprising only samples from sites 8 and 9 (Azizakouè 1 and Azizakouè 2), was characterized by fine sediment particles and high salinity. Group V (ah2) mostly comprises samples from the Aho channel (sites 11, 12 and 13) with high salinity, great depths, and sandy-muddy sediments lined with mangroves trees.

\section{Faunal composition}

A total of 4,179 benthic macroinvertebrates were collected with a grab during the sampling. Altogether, 182 taxa, belonging to 25 orders and 114 families were recorded. The fauna comprises essentially two zoological groups which make up $81.45 \%$ of the families and $79.12 \%$ of species identified (Figure 4). Molluscs (47 taxa), Insects (61 taxa), and Crustaceans (38 taxa) were found. Among the richest families were the Thiaridae (Molluscs Gastropoda) and the Chironomidae (Insects Diptera) with 7 taxa and the Nereidae (Polychaeta Nereidiformia) with 5 taxa. The Neritidae (Molluscs Gastropoda); the Coenagrionidae (Insects Odonata) and the Dytiscidae (Insects Coleoptera) had 4 taxa. The list of macroinvertebrate organisms collected in the Coastal Lagoon during the study is shown in Table 3.

Table 2 shows the abundance of the zoological groups encountered during the study. The Molluscs with $39.51 \%$ of the total abundance were the main group of this ecosystem. There were followed by Crustaceans that represented $31.6 \%$ of the total number of individuals. The Insects represented $13.47 \%$ of this abundance. The Gastropoda with $33.98 \%$ of the total abundance was the most encountered order. It was followed by the Decapoda (13.95\%), the Amphipoda $(12.17 \%)$, the Nereidiformia $(7.18 \%)$, the Hirudinea $(5.84 \%)$ and the Bivalvia (5.53\%) respectively. 
Seven families were predominant, Molluscs, 3 (Potamididae: $254.81 \mathrm{ind} / \mathrm{m}^{2}$; Thiaridae: $211.36 \mathrm{ind} / \mathrm{m}^{2}$; Neritidae: 123.51 ind $/ \mathrm{m}^{2}$ ); Crustaceans, 3 (Diogenidae: 178.76 ind $/ \mathrm{m}^{2}$; Melitidae: $183.70 \mathrm{ind} / \mathrm{m}^{2}$ and Aoridae: $159.01 \mathrm{ind} / \mathrm{m}^{2}$ ), and Polychaeta, 1 (Nereidae: $123.45 \mathrm{ind} / \mathrm{m}^{2}$ ). Other families such as the Chironomidae, the Municidae, the Gammaridae and the Dytiscidae had their abundance comprised between $4 \%$ and $2 \%$ of the total of the organisms.

The highest abundance of the Gastropoda is due to a few species like Tympanotonus $f$. radula $\left(464.20 \mathrm{ind} / \mathrm{m}^{2}\right)$, Pachymelania aurita (366.42 ind $\left./ \mathrm{m}^{2}\right)$, Neritina glabrata and Hydrobia guyenoti. Other species among which Clibanarius spp. (178.76 ind $\left./ \mathrm{m}^{2}\right)$, Nereis sp. (132.34 ind $\left./ \mathrm{m}^{2}\right)$, Quadrivisio sp. (124.44 ind $\left./ \mathrm{m}^{2}\right)$ and Chironomus sp. (117.74 ind $\left./ \mathrm{m}^{2}\right)$ also presented a higher abundance. About $65 \%$ of the benthic organisms had less than $5 \%$ of the total abundance.

\section{Community structure}

Figure 5 was used to illustrate the variations of the taxonomic richness, relative abundance, Shannon index H' and evenness of the taxa of each group of sites defined by the typology. Taxonomic richness increased from downstream up. The upstream part of the lagoon was rich with a total of 77 taxa collected at gal, 65 at ah2 representing respectively 971 and 844 individuals of benthic animals collected respectively in these groups. The group oal had the highest abundance (1,143 individuals) and 66 taxa. In the downstream part, respectively 51 and 55 taxa were sampled at od 2 and to 1 and thus had the lowest abundances (526 and 581 individuals). No significant difference (Kruskal-Wallis test: $\mathrm{p}>0.05$ ) was noted between taxonomic richness of groups of sites.

Figure 6 presented the important taxa in each group of sites. In all groups, the Gastropoda order was the most abundant taxa determined by the proliferation of Tympanotonus fuscatus radula. It was followed by Pachymelania aurita, Pachymelania fusca, Hydrobia guyenoti, and Neritina glabrata. Some taxa were specific to each group and can be encountered only in those groups.

In the group ot 1 determined by depth and percentage of clay and less to oxygen, Diptera and Haplotaxida taxa were identified with high abundance. In addition, there were Chironomidae (Chironomus sp., Polypedilum sp.), Chrysomelidae (Donacia sp.), Potamididae (Tympanotonus fuscatus radula), Hydrobiidae (Hydrobia guyenoti) and Tubificidae (Tubifex sp.) and other worms.

In the channel ah2 determined by muddy sediments and high depth and salinity values, Corbula trigona and the Nereis sp. were the most taxa collected after the Gastropoda species. The abundance of this Bivalvia decreased considerably over the sampling period. In the middle sites (near the mouth of the Lagoon) determined by salinity gradient, water transparency and depth, with a substrate muddy-sandy, the Decapoda (Callinectes amnicola, C. sapidus, Clibanarius spp.), the Coleoptera (Dyticus sp.), the Amphipoda (Gammarus sp.), the Polychaeta (Nereis sp., N. diversicolor, Dodecaria sp., Nephthys sp.) and the Hirudinea (Erpobdellidae) were more abundant. The upper sites gal, strongly related to dissolved oxygen and percentage of sand, was characterized by the high abundance of Baetidae (Baetis sp.), Coenagrionidae, Aoridae (Grandiddierella africana), Melitidae (Quadriviso sp.), $\quad$ Paleomonidae (Macrobrachium spp.), Thiaridae (Pachymelania aurita, Pachymelania fusca) and Nereidae (Nereis sp., N. diversicolor).

The Shannon index values ranged between 1.29 bits at ah2 and 3.84 bits at gal in the upper part of the lagoon but the group ot 1 has registered the low values of this index. The evenness value was superior to 0.5 in all groups of sites except in ah2 (0.27). These indexes did not have significant difference (Mann-Whitney, $\mathrm{p}>0.05$ ) between groups of sites. 


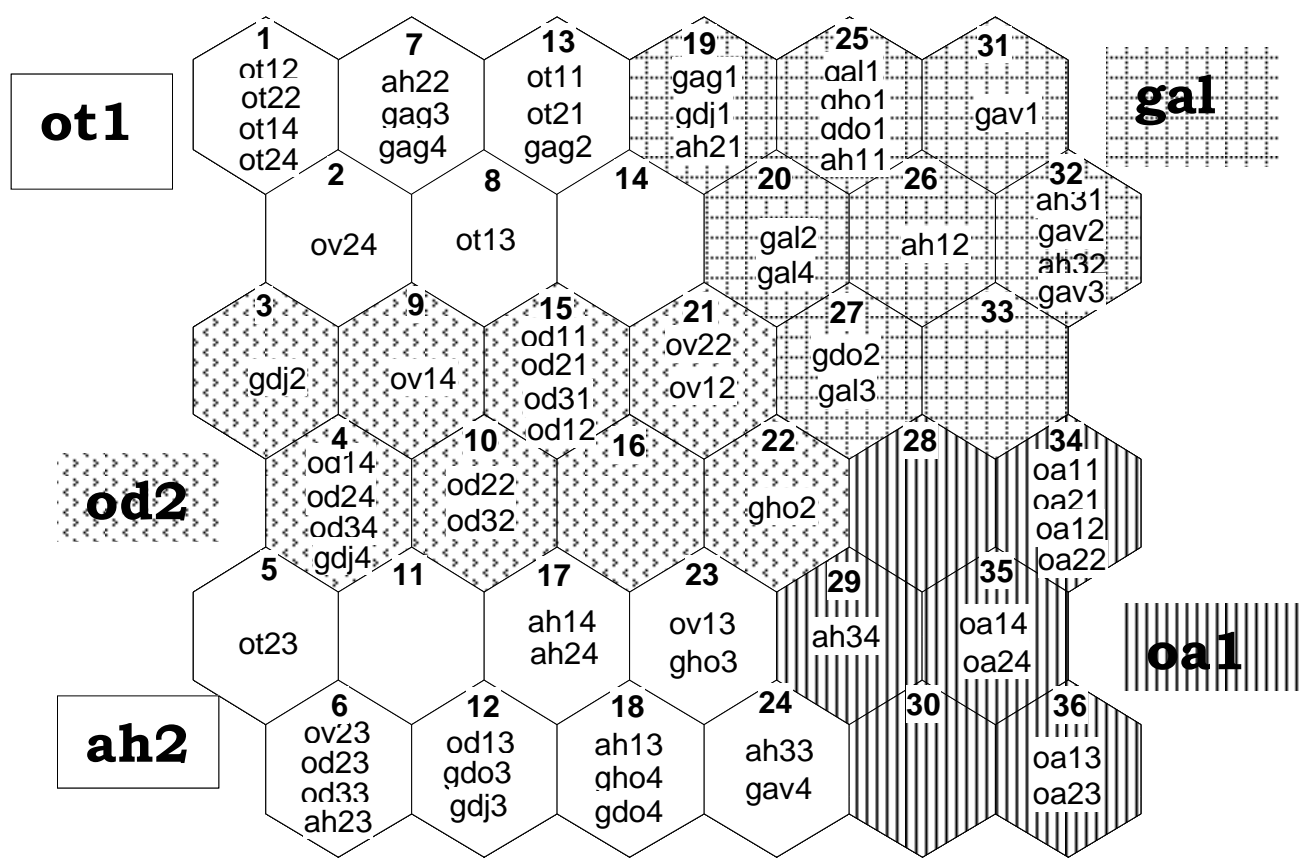

Figure 2: SOM map of the samples classification on the basis of environmental variables. ot 1 , ot 2 and so on are the samples sites, the second number near the samples sites is the number of the sampling season and the number of each cell is in bold.

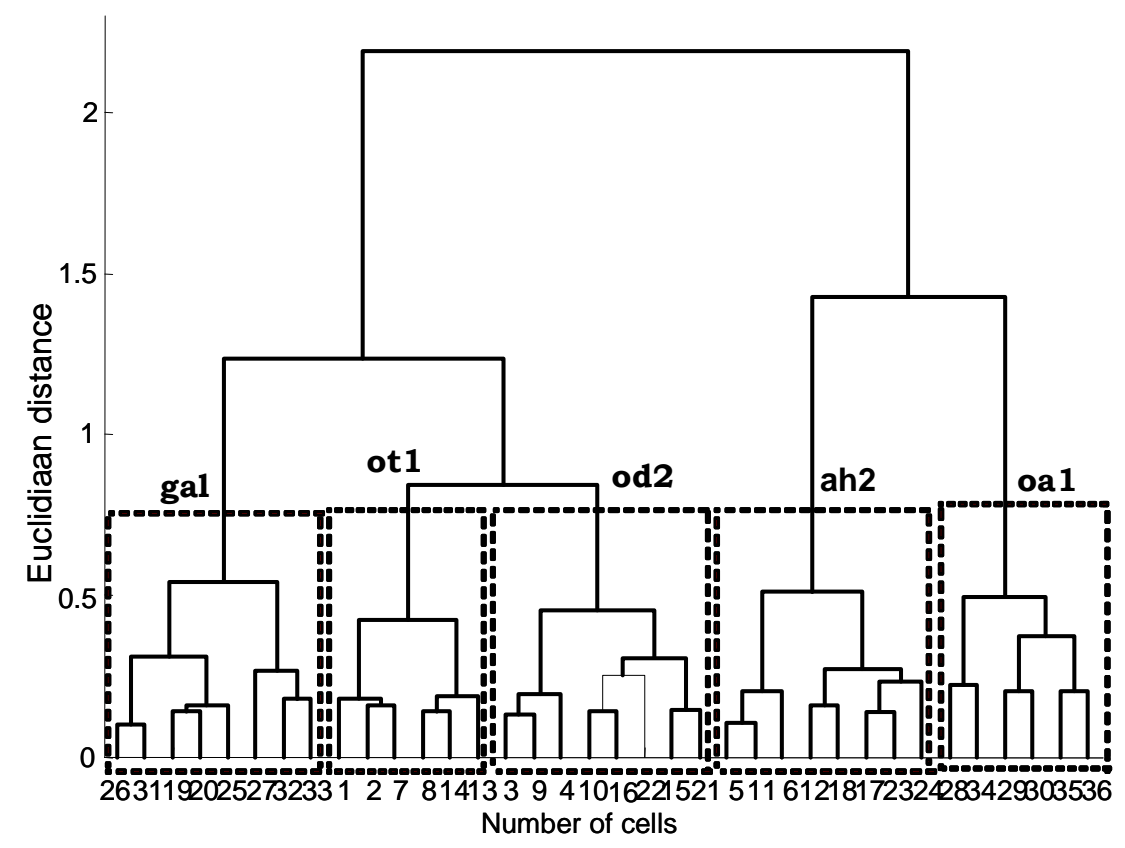

Figure 3: Hierarchical clustering of the SOM cells with a Ward linkage method and a Euclidian distance. 


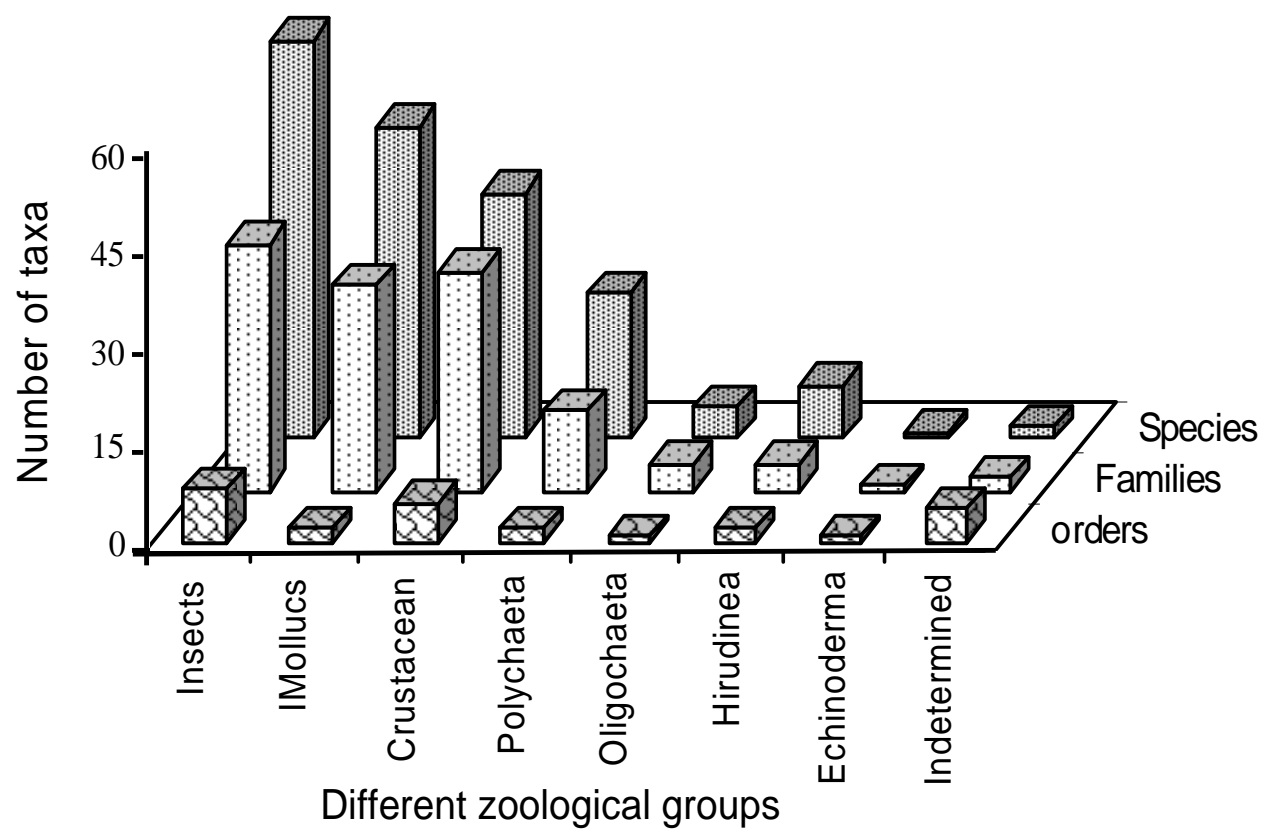

Figure 4: Composition of the macroinvertebrates fauna during the sampling period of the Coastal Lagoon.
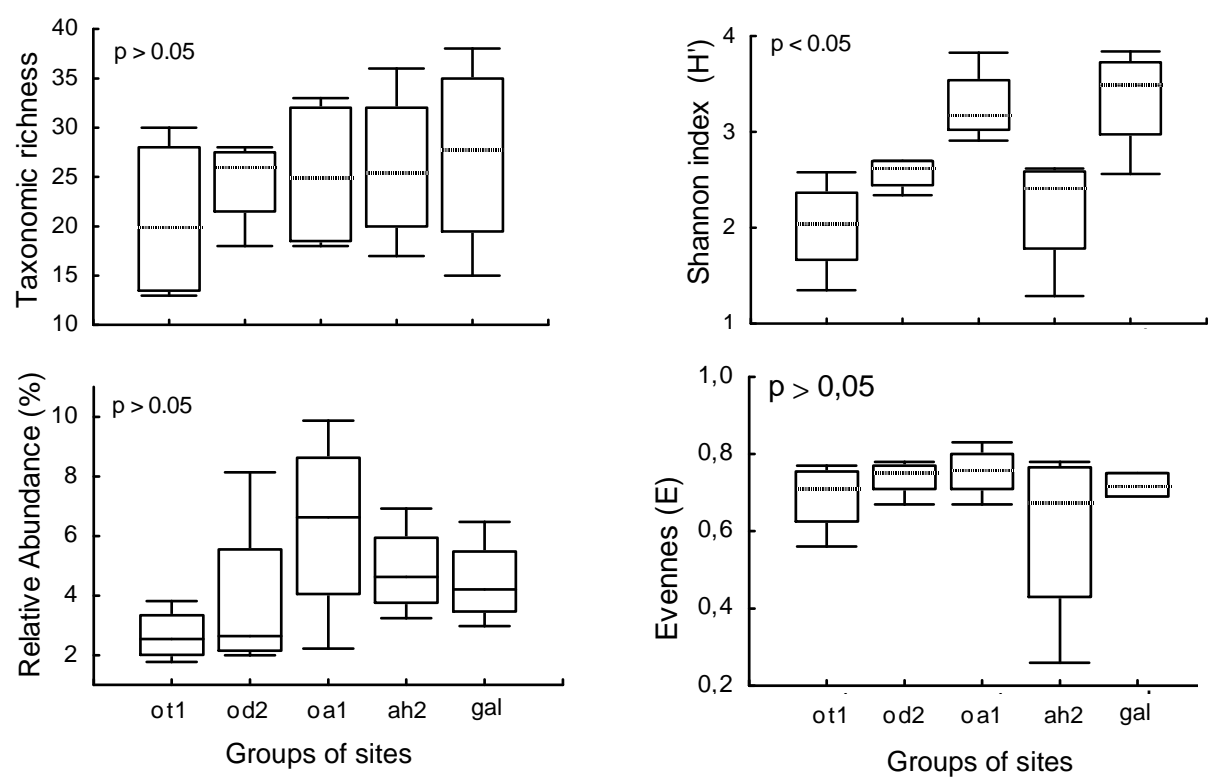

Figure 5: Variations of taxonomic richness, relative abundance, Shannon index and evenness in the groups of sites. 


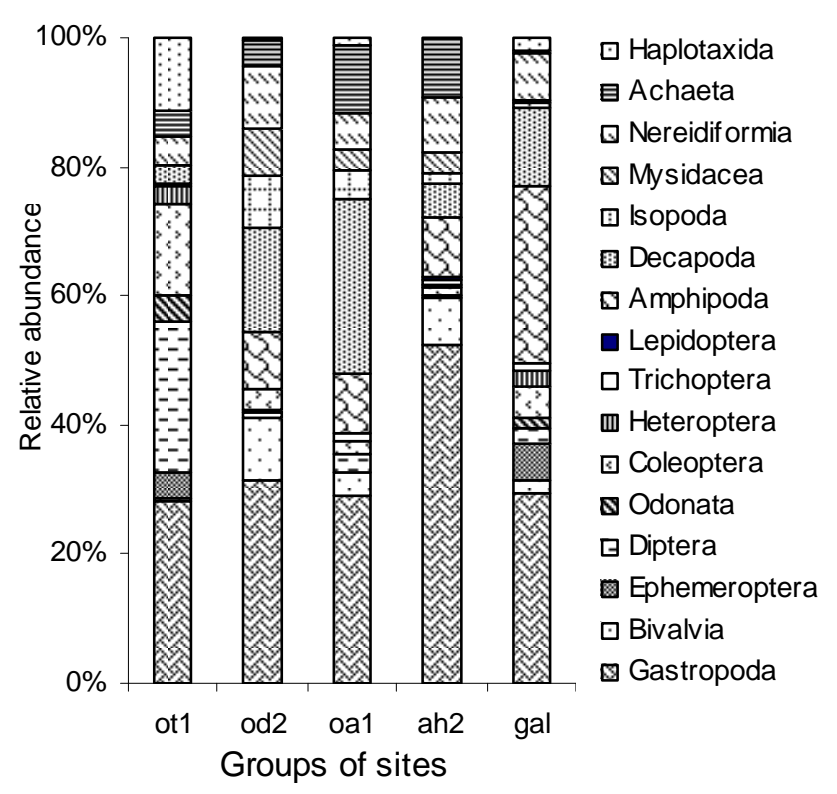

Figure 6: Relative abundance of benthic organisms in each group of sites.

Table 1: Mean and range values of environmental variables in the lagoon and analysis of variance on data (Kruskal-Wallis test).

\begin{tabular}{lccc}
\hline Variables & Mean (range) values & H & p \\
\hline Depth (m) & $1.25(0.15-2.8)$ & 6.43 & 0.0928 \\
Salinity (psu) & $5.28(0-26.6)$ & 28.62 & $<0.0001 *$ \\
Dissolved Oxygen $\left(\mathrm{mg} . \mathrm{l}^{-1}\right)$ & $5.37(0.40-11.2)$ & 27.49 & $<0.0001 *$ \\
Transparency (cm) & $37,11(10-100)$ & 35.12 & $<0.0001 *$ \\
Sand (\%) & $70.24(2.91-95.47)$ & - & - \\
Silt-clay (\%) & $27.32(2.97-97.02)$ & - & - \\
\hline \multicolumn{2}{r}{ Numbers in parenthesis represent the minimum and the maximum values. Significant difference $=*$} \\
\end{tabular}

Table 2: Abundance of the zoological groups collected in the lagoon.

\begin{tabular}{lcc}
\hline Zoological groups & Abundance & Relative abundance (\%) \\
\hline Molluscs & 1651 & 39.51 \\
Insects & 563 & 13.47 \\
Crustaceans & 1323 & 31.66 \\
Polychaeta & 300 & 7.18 \\
Hirudinea & 245 & 5.86 \\
Oligochaeta & 97 & 2.32 \\
\hline
\end{tabular}


Table 3: List of macroinvertebrate organisms collected in the Coastal Lagoon during the study.

\begin{tabular}{|c|c|c|c|c|c|c|c|c|c|c|c|c|c|c|c|c|c|c|c|}
\hline \multirow{2}{*}{\multicolumn{2}{|c|}{$\begin{array}{l}\text { TAXA } \\
\text { CRUSTACEANS }\end{array}$}} & \multicolumn{18}{|c|}{ Sampling sites } \\
\hline & & 1 & 23 & 32 & 4 & 5 & 6 & 7 & 8 & 9 & 11 & 12 & 13 & 10 & 15 & 14 & 17 & 16 & 18 \\
\hline \multicolumn{20}{|l|}{ AMPHIPODA } \\
\hline Gammaridae & Gammarus Linne, 1758) sp. & & & & * & $*$ & $*$ & $*$ & $*$ & & $*$ & $*$ & & $*$ & $*$ & & $*$ & $*$ & $*$ \\
\hline Photidae & Photis sp. & & & * & & & & $*$ & & & $*$ & & & & $*$ & & * & $*$ & $*$ \\
\hline Metilidae & Quadrivisio sp. & $*$ & & $*$ & * & $*$ & $*$ & $*$ & $*$ & & $*$ & $*$ & $*$ & $*$ & $*$ & $*$ & $*$ & $*$ & $*$ \\
\hline Aoridae & Grandidierella africana & $*$ & & & & $*$ & $*$ & $*$ & $*$ & & $*$ & $*$ & & & $*$ & $*$ & $*$ & $*$ & $*$ \\
\hline \multicolumn{20}{|l|}{ DECAPODA } \\
\hline $\begin{array}{l}\text { Diogenidae } \\
\text { Potamonidae } \\
\text { Xantidae }\end{array}$ & $\begin{array}{l}\text { Clibanarius (Latreille, 1818) spp. } \\
\text { Potamon ibericum (Czerniavsky, } \\
\text { Heteropanope africanus (De Man, }\end{array}$ & $*$ & & $*$ & * & $*$ & $*$ & $*$ & $*$ & $*$ & $\begin{array}{l}* \\
*\end{array}$ & $*$ & $*$ & $*$ & $*$ & $*$ & $\begin{array}{l}* \\
*\end{array}$ & $*$ & $*$ \\
\hline \multirow{2}{*}{ Paléomonidae } & Macrobrachium sp. & & & & * & * & * & & * & * & & & $*$ & & * & * & * & $*$ & * \\
\hline & Macrobrachium vollenhovenii & & & & & $*$ & & & $*$ & $*$ & & & $*$ & & & $*$ & $*$ & $*$ & $*$ \\
\hline \multirow{4}{*}{ Sesarmidae } & Sesarma luzardi (Desmarest 1825) & & & & & & & & & & & $*$ & $*$ & & & $*$ & $*$ & $*$ & $*$ \\
\hline & Sesarma sp. & & & & & & & & & $*$ & $*$ & $*$ & & & & $*$ & $*$ & $*$ & $*$ \\
\hline & Sesarma angolense & & & & & & & & & & & $*$ & & & & & & & \\
\hline & Sesarma elegans (Warner 1967) & & & & & & & & & * & $*$ & $*$ & & * & * & * & * & * & $*$ \\
\hline Ocypodidae & Uca tangeri (Eydoux, 1835$)$ & & & & & & & & & $*$ & & & & & & & & & \\
\hline \multirow{2}{*}{ Grapsidae } & Pachygrapsus gracilis (Saussure, & & & & & & & & & * & $*$ & * & & & & $*$ & & & \\
\hline & Goniopsis pelii & & & & & & & & & & $*$ & * & & & & & & & \\
\hline \multirow{3}{*}{ Portunidae } & Callinectes amnicola $(\mathrm{De}$ & & & $* *$ & * & & & & * & $*$ & $*$ & $*$ & * & * & & * & & & \\
\hline & Callinectes danae (Smith, 1869) & & & $*$ & * & & & & $*$ & $*$ & $*$ & & & $*$ & & & & & \\
\hline & Callinectes sapidus (Rathburn,1896). & & & & $*$ & & & & $*$ & $*$ & & & * & $*$ & & & & & \\
\hline Gecarcinidae & Cardiosoma armatum (Herklots, & & & * & & & & & $*$ & $*$ & & & $*$ & & & & & * & * \\
\hline Palinuridae & Panulirus sp. & & & & & & & & & & & & & $*$ & $*$ & * & & & \\
\hline Callianassidae & Callianassa sp. & & & & & & & & & & & & & & & $*$ & & & \\
\hline Atyidae & Atyaephyra sp. & & & $*$ & * & * & & & * & & & * & & & $*$ & * & * & & \\
\hline Leptotracae & Nebalia sp. & & & & & & & & & & & & & & $*$ & $*$ & & $*$ & \\
\hline Hypolitidae & & & & & * & & & & & & & & & $*$ & $*$ & & & & \\
\hline Peneaeidae & Penaeus sp. & & & & & & & & $*$ & $*$ & & $*$ & $*$ & $*$ & $*$ & $*$ & $*$ & $*$ & $*$ \\
\hline
\end{tabular}


D. ADANDEDJAN et al. / Int. J. Biol. Chem. Sci. 6(3):1233-1252, 2012

\begin{tabular}{|c|c|c|c|c|c|c|c|c|c|c|c|c|c|c|c|c|c|c|c|}
\hline \multicolumn{20}{|l|}{ ISOPODA } \\
\hline Cassidinidae & Cassidinidea $\mathrm{sp}$. & $*$ & & & $*$ & & & & & & $*$ & & & & & & & & \\
\hline Cirolanidae & Excirolana latipes (Bernard, 1914) & & & $*$ & & $*$ & $*$ & & $*$ & & $*$ & $*$ & & & & & & $*$ & \\
\hline \multirow{2}{*}{ Anthuridae } & Cyathura sp. & & & & $*$ & & & & & & & & & $*$ & $*$ & $*$ & & & \\
\hline & Anthura gracilis (Montagu, 1808) & & & & $*$ & & & & $*$ & & & & & & & $*$ & & & \\
\hline Sphaeromatidae & Sphaeroma terebrans (Bate, 1866$)$ & $*$ & $*$ & $*$ & $*$ & & $*$ & $*$ & $*$ & $*$ & $*$ & * & & & & & $*$ & $*$ & $*$ \\
\hline Municidae & Iromura powerly (Kensley, 1980) & & & & & $*$ & $*$ & $*$ & $*$ & & & $*$ & & & & $*$ & & & \\
\hline \multicolumn{20}{|l|}{ MYSIDACEA } \\
\hline Mysidae & & & & $*$ & $*$ & $*$ & $*$ & $*$ & $*$ & $*$ & $*$ & & & & & & $*$ & $*$ & \\
\hline \multicolumn{20}{|l|}{ TANAIDACEA } \\
\hline Tanaidae & Tanais dulongi (Audouin, 1826) & & & & & & $*$ & & & & & & & & & & & & \\
\hline \multicolumn{20}{|l|}{ CIRRIPEDA } \\
\hline \multirow{2}{*}{ Balanidae } & Chthamalus rhizophora (Pilsbry, & & & & $*$ & & & $*$ & $*$ & $*$ & $*$ & $*$ & $*$ & $*$ & $*$ & $*$ & $*$ & $*$ & \\
\hline & Balanus sp. & & & & & $*$ & $*$ & $*$ & $*$ & $*$ & $*$ & $*$ & $*$ & $*$ & $*$ & $*$ & & & \\
\hline Autres & & & & & $*$ & $*$ & $*$ & $*$ & $*$ & $*$ & & & & * & * & * & $*$ & $*$ & $*$ \\
\hline \multicolumn{20}{|l|}{ MOLLUSCS } \\
\hline \multicolumn{20}{|c|}{ GASTROPODA } \\
\hline \multicolumn{20}{|c|}{ MESOGASTROPODA } \\
\hline Ampullariidae & Lanistes variscus (Müller, 1774) & $*$ & & & & & & & & & & & & & & & & & $*$ \\
\hline Bithyniidae & Gabbiella africana (Frauenfeld, & & & & & & & & & & & & & & & & & $*$ & $*$ \\
\hline Carditidae & Cardita calyculata (Broderip \& & & & & & & & & & & & & & & & $*$ & & & \\
\hline Hydrobiidae & Hydrobia guyenoti (Binder, 1955) & $*$ & $*$ & & & $*$ & $*$ & $*$ & $*$ & & & $*$ & & & & & $*$ & $*$ & $*$ \\
\hline \multirow[t]{2}{*}{ Littorinida } & Littorina scabra (Linna eus, 1758) & & & $*$ & & & & & $*$ & & & & & $*$ & & & & & \\
\hline & Littorina africana (Farassac, 1921) & & & $*$ & & & & & & & $*$ & $*$ & * & $*$ & * & & & & \\
\hline Lucinidae & Codakia orbicularis (Linne, 1758) & & & & & & $*$ & & & & & & & & & & & & \\
\hline Thiaridae & Pachymelania aurita (Müller, 1872) & & & $*$ & $*$ & $*$ & $*$ & $*$ & $*$ & $*$ & $*$ & $*$ & $*$ & $*$ & $*$ & $*$ & $*$ & $*$ & $*$ \\
\hline
\end{tabular}

N.B. : $1=$ ot $1 ; 2=$ ot $2 ; 3=$ ov $1 ; 4=$ ov $2 ; 5=$ od $1 ; 6=$ od $2 ; 7=$ od $3 ; 8=$ oa $1 ; 9=$ oa $2 ;$

$10=$ gdj $; 11=$ ah $1 ; 12=$ ah2 $; 13=$ ah $3 ; 14=$ gho. $; 15=$ gdo $; 16=$ gal $; 17=$ gav $; 18=$ gag 


\section{ADANDEDJAN et al. / Int. J. Biol. Chem. Sci. 6(3):1233-1252, 2012}

\begin{tabular}{|c|c|c|c|c|c|c|c|c|c|c|c|c|c|c|c|c|c|c|c|}
\hline \multirow[t]{2}{*}{ TAXA } & \multirow[b]{2}{*}{ GASTROPODA } & \multicolumn{18}{|c|}{ Sampling sites } \\
\hline & & 1 & 2 & 3 & 4 & 5 & 6 & 7 & 8 & 9 & 911 & 12 & 13 & 10 & 15 & 14 & 17 & 16 & 18 \\
\hline \multicolumn{20}{|c|}{ MESOGASTROPOA } \\
\hline \multirow{6}{*}{ Thiaridae } & Pachymelania f. quadriseriata & & & * & $*$ & & & & * & * & & & $*$ & $*$ & & $*$ & $*$ & $*$ & $*$ \\
\hline & Pachymelania byronensis (Gray, & & & & & & & & & & & & & & & & & $*$ & $*$ \\
\hline & Pachymelania fusca (Gemlin, & & & & & & & & & & & & & & & & $*$ & $*$ & $*$ \\
\hline & Melanoides tuberculata (Müller, & & $*$ & * & & & $*$ & * & & & & $*$ & & & & & & $*$ & $*$ \\
\hline & Melanoides anomala (Smith, 1877) & $*$ & & & & & & & & & & & & & & & & $*$ & \\
\hline & Potadoma sp. & & & $*$ & & & & & & & $*$ & & & & & & & $*$ & \\
\hline \multirow{2}{*}{ Muricidae } & Thais nodosa (Linnaeus, 1758) & & & & & & & & & & & & & $*$ & $*$ & & & & \\
\hline & Thais coronata (Lamarck, 1822) & & & & & & & & & & & & & * & $*$ & & & & \\
\hline Naticidae & Polinices (Montfort, 1810) sp. & & & & & & & & & & & & & & & & & $*$ & \\
\hline \multirow{4}{*}{ Neritidae } & Neritina cristata (Morelet, 1858) & $*$ & $*$ & & & & & & & & & & & & & & & & \\
\hline & Neritina glabrata (Sowerby, 1849) & & & $*$ & $*$ & $*$ & $*$ & $*$ & * & * & $* *$ & $*$ & $*$ & $*$ & $*$ & $*$ & $*$ & $*$ & \\
\hline & Neritina afra (Sowerby, 1841) & & & $*$ & $*$ & & & & & & & & & & & $*$ & & & \\
\hline & Neritina kuramoensis (Yoloye \& & & & $*$ & $*$ & & & & & * & $* *$ & $*$ & & $*$ & & $*$ & $*$ & $*$ & \\
\hline \multirow{2}{*}{ Potamididae } & Tympanotonus f. radula (Linné, & $*$ & & $*$ & $*$ & $*$ & $*$ & $*$ & * & * & * * & $*$ & $*$ & $*$ & $*$ & $*$ & $*$ & $*$ & $*$ \\
\hline & Turitella sp. & * & & & & * & $*$ & & * & & & $*$ & & $*$ & $*$ & & & & \\
\hline Indetermined & Indetermined & & & & & & & & & & & & & & & & & $*$ & $*$ \\
\hline \multicolumn{20}{|c|}{ BASOMATOPHORA } \\
\hline Lymnaeidae & Lymnaea sp. & & & & & & & & & & & & & & & & & & \\
\hline \multirow{2}{*}{ Planorbidae } & Indoplanorbis $\mathrm{sp}$. & $*$ & $*$ & & & $*$ & & & * & & & & & & & & & $*$ & \\
\hline & Giraulus sp. & $*$ & $*$ & & * & & & & & & & & & & & & & & $*$ \\
\hline Physidae & Physa marmorata (Guilding, 1828) & $*$ & $*$ & & & & & & * & 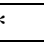 & & & & & & & $*$ & $*$ & \\
\hline \multicolumn{20}{|c|}{ PROSOBRANCHES } \\
\hline Patellidae & Patella (Linnaeus, 1758) sp. & * & $*$ & & $*$ & & & & $*$ & 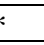 & & & & & & & & & \\
\hline Haliotidae & Haliotis sp. & & & & & & $*$ & & & & & & & $*$ & & & & & \\
\hline \multicolumn{20}{|c|}{ POLYPLACOPHORA } \\
\hline & Chiton (Gray, 1821) & & $*$ & & & & & & & & & $*$ & $*$ & $*$ & $*$ & & & & \\
\hline \multicolumn{20}{|c|}{ EULAMELLIBRANCHS } \\
\hline Arcidae & Anadara senilis (Linnaeus, 1758) & & & & & & & & * & " & & & & $*$ & $*$ & & & & \\
\hline
\end{tabular}


D. ADANDEDJAN et al. / Int. J. Biol. Chem. Sci. 6(3):1233-1252, 2012

\begin{tabular}{|c|c|c|c|c|c|c|c|c|c|c|c|c|c|c|c|c|c|c|}
\hline Ostreidae & Cassostrea sp. & * & & * & * & * & & * & $*$ & & $*$ & $*$ & * & $*$ & & * & * & \\
\hline \multirow{2}{*}{ Unionidae } & Unio crassus (Philipsson, 1788) & & * & & & & & & $*$ & & & & & & & & & \\
\hline & Potomida littoralis (Jacquemin, & $*$ & * & * & * & * & $*$ & * & * & $*$ & $*$ & $*$ & $*$ & & & $*$ & $*$ & $*$ \\
\hline Corbulidae & Corbula trigona (Hinds, 1843 ) & & & * & $*$ & $*$ & & $*$ & $*$ & $*$ & $*$ & $*$ & $*$ & $*$ & $*$ & $*$ & $*$ & $*$ \\
\hline Dreissenidae & Congeria chochleata (Van & & * & & & & & & & & & & * & $*$ & * & & * & \\
\hline \multirow{2}{*}{ Tellinidae } & Tellina (Linnaeus, 1758) sp. & & & * & & * & & & & $*$ & & & & $*$ & & * & & $*$ \\
\hline & Tellina ampullicea (Philippi, 1844) & & & * & * & * & $*$ & * & & & $*$ & & $*$ & * & $*$ & * & & \\
\hline \multirow{2}{*}{ Sphaeriidae } & Pisdium sp. & & & & * & & & * & & & & & & & & & & \\
\hline & Sphaerium sp. & & * & & * & & & * & $*$ & & & & & & & & & \\
\hline Mactridae & Mactra (Linnaeus 1758) sp. & & & & & & & & & * & & & & & & & & \\
\hline Donacidae & Iphigenia (Schumacher, 1817) sp. & & & * & & & & * & & & & & $*$ & & & & & \\
\hline \multirow{2}{*}{ Solecurtidae } & Pharus legumen (Linnaeus, 1758) & & & * & & & & & & & & & $*$ & & $*$ & & & \\
\hline & Tagelus angulatus (Sowerby, 1874) & & & * & & & & & & & & & $*$ & & & & & \\
\hline Mytilidae & Brachyodontes (Pilsbry, 1921) sp. & \% & 赵 & & & & & * & $*$ & & $*$ & & $*$ & $*$ & & & & \\
\hline Veneridae & Pitar tumens (Gmelin, 1791) & & 赵 & * & & & & & & & * & & & $*$ & & & & \\
\hline \multicolumn{19}{|l|}{ INSECTS } \\
\hline & EPHEMEROPTERA & & & & & & & & & & & & & & & & & \\
\hline \multirow{2}{*}{ Ephemeridae } & Ephemera (Linné, 1758) sp. & & & & & & & & & & & & & & & & * & \\
\hline & Eatonica crassi (McCafferty. 1971) & & & & & & & & & & & & & & & & * & $*$ \\
\hline \multirow{3}{*}{ Baetidae } & Baetis (Leach, 1815) sp. & & & & & & & & & & & & & & & * & * & * \\
\hline & Pseudocloeon sp. & & & & & & & & & & & & & & & & * & \\
\hline & Centroptilum (Eaton, 1869) sp. & * & & & & & & & & & & & & & & & * & \\
\hline Leptophlebiidae & Thraulus (Eaton, 1881) sp. & + & & & & & & & & & & & & & & & * & * \\
\hline \multirow{2}{*}{ Heptageniidae } & Afronurus (Lestage, 1924) sp. & & & & & & & & & & & & & & & & $*$ & $*$ \\
\hline & Notonurus sp. & & * & & & & & & & & & & & & & & & * \\
\hline
\end{tabular}

N.B. $: 1=$ ot $1 ; 2=$ ot $2 ; 3=$ ov $1 ; 4=$ ov $2 ; 5=$ od $1 ; 6=$ od $2 ; 7=$ od $3 ; 8=$ oa $1 ; 9=$ oa $2 ;$

$10=$ gdj $; 11=$ ah $1 ; 12=$ ah $2 ; 13=$ ah $3 ; 14=$ gho $; 15=$ gdo $; 16=$ gal $; 17=$ gav $; 18=$ gag . 


\begin{tabular}{|c|c|c|c|c|c|c|c|c|c|c|c|c|c|c|c|c|c|c|c|}
\hline \multirow{2}{*}{\multicolumn{2}{|c|}{$\begin{array}{l}\text { TAXA } \\
\text { HETEROPTERA-HEMIPTERA }\end{array}$}} & \multicolumn{18}{|c|}{ Sampling sites } \\
\hline & & 1 & 2 & 3 & 4 & 5 & 6 & 7 & 8 & 9 & 11 & 12 & 13 & $\mathbf{1 0}$ & 15 & 14 & 17 & 16 & 18 \\
\hline \multirow{2}{*}{ Belostomidae } & Diplonychus sp. & & $*$ & & & & & & & & & & & & & & $*$ & $*$ & $*$ \\
\hline & Plea sp. & $*$ & $*$ & & & & & & & & & & & & & & & & \\
\hline \multirow{2}{*}{ Gerridae } & Limnogonus & & $*$ & & & & & $*$ & & & & & & & & & & $*$ & $*$ \\
\hline & Eurymethra sp. & $*$ & $*$ & & & & & & & & & & & & & & & & \\
\hline Veliidae & Microvelia sp. & & & & & & & & & & & & & & & & & & $*$ \\
\hline Nepidae & Nepa (Poisson, 1951) sp. & $*$ & & $*$ & & & & & & & & & & & & & & $*$ & $*$ \\
\hline Ranatridae & Ranatra (Poisson, 1965) sp. & & & $*$ & & & & & & & & & & & & & & $*$ & $*$ \\
\hline Agriotypidae & Agriotypus sp. & & & & & & & & & & & & & & & $*$ & & & \\
\hline Corixidae & Micronecta sp. & $*$ & $*$ & & & & & & & & & & & & & & & & \\
\hline Hydrometridae & Hydrometra (Linnaeus 1758) & $*$ & & & & & & & & & & & & & & & & & \\
\hline \multirow{2}{*}{ Naucoridae } & Naucoris cinicoides & & & & & & & & & & & & & & & & & $*$ & \\
\hline & PLECOPTERA & & & & & & & & & & & & & & & & & & \\
\hline \multirow[t]{2}{*}{ Capniidae } & Capnia sp. & & & & & & & & & & & & & & & & & $*$ & $*$ \\
\hline & ODONATA & & & & & & & & & & & & & & & & & & \\
\hline \multirow{3}{*}{ Libellulidae } & Libella sp. & $*$ & $*$ & $*$ & & & & & & & & $*$ & & & & & & & \\
\hline & Libellula sp. & $*$ & $*$ & & * & & & & & & & $*$ & & & & & $*$ & $*$ & $*$ \\
\hline & Palpopleura lucia lucia (Dnq, & & $*$ & & & & & & & & & & & & & & & & \\
\hline \multirow{3}{*}{ Corduliidae } & Phyllomacromia sp. & $*$ & & & & & & & & & & & & & & & & & \\
\hline & Cordulia sp. & & $*$ & & & & & & & & & & & & & & & $*$ & \\
\hline & Argia vivida & $*$ & & & & & & & & & & & & & & & & & \\
\hline \multirow{3}{*}{ Coenagrionidae } & Coenagrion spp. & & $*$ & & & $*$ & & & & $*$ & & & & & & & & & \\
\hline & Pseudagrion wellani & $*$ & & & & & & & $*$ & & & & & & & & & $*$ & \\
\hline & Ischnura sp. & & $*$ & & & & & & & & & & & & & & & & \\
\hline \multirow[t]{2}{*}{ Calopterygidae } & Phaon iridipennis & $*$ & & & & & & & & & $*$ & & & & & & & $*$ & \\
\hline & DIPTERA & & & & & & & & & & & & & & & & & & \\
\hline Ceratopogonidae & Ceratopogon $\mathrm{sp}$. & & & & & & & & & & & & & & & & & $*$ & $*$ \\
\hline \multirow{2}{*}{ Chironomidae } & Chironomus sp. & $*$ & $*$ & $*$ & & $*$ & $*$ & & & & $*$ & $*$ & & & & $*$ & $*$ & $*$ & \\
\hline & Chironomus formosipennis & $*$ & $*$ & & $*$ & & & $*$ & & $*$ & $*$ & & & & & & & & \\
\hline
\end{tabular}


D. ADANDEDJAN et al. / Int. J. Biol. Chem. Sci. 6(3):1233-1252, 2012

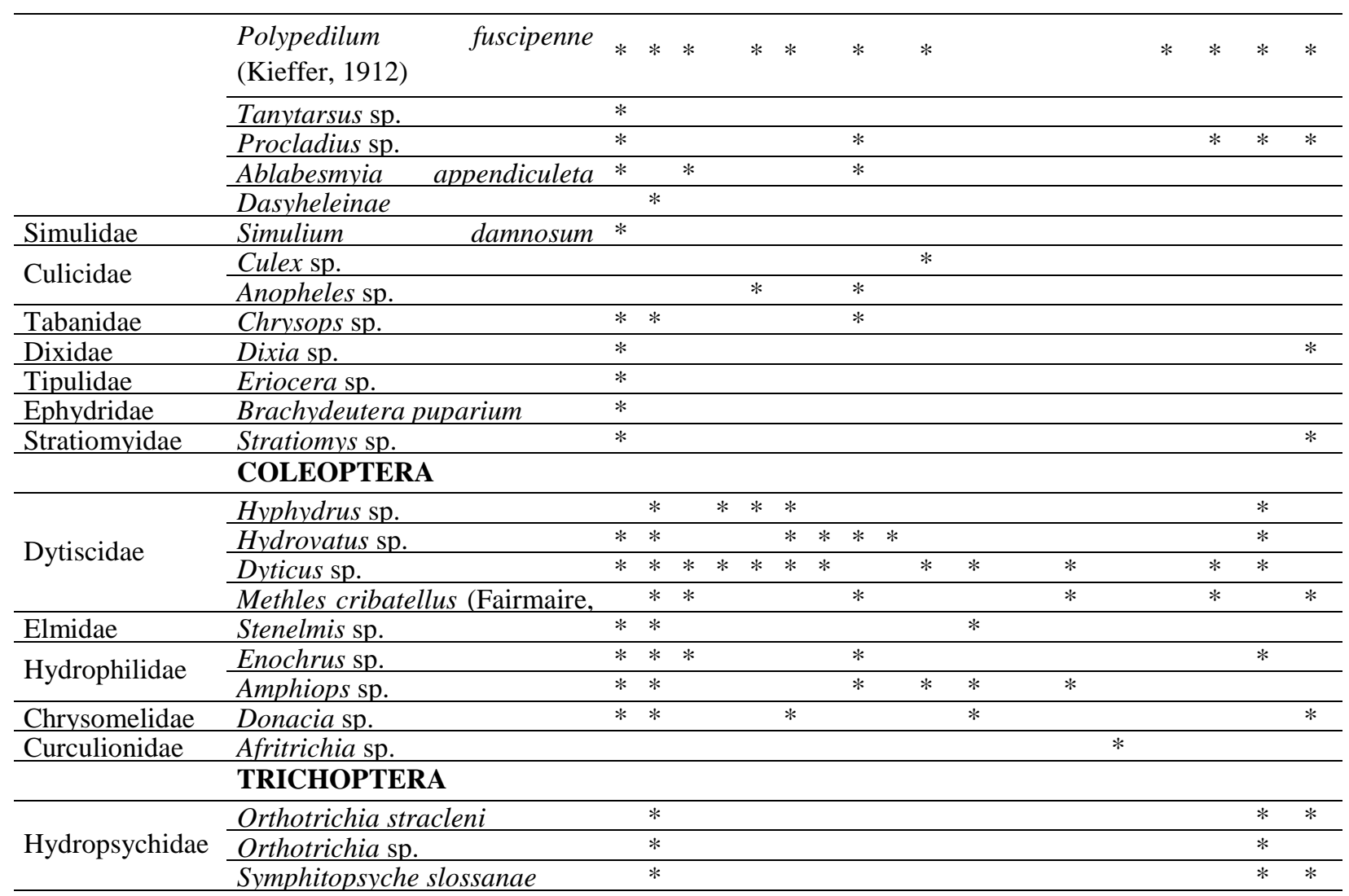

N.B $: 1=$ ot $1 ; 2=$ ot $2 ; 3=$ ov $1 ; 4=$ ov $2 ; 5=$ od $1 ; 6=$ od $2 ; 7=$ od $3 ; 8=$ oa $1 ; 9=$ oa 2 ;

$10=$ gdj; $11=$ ah $1 ; 12=$ ah $2 ; 13=$ ah $3 ; 14=$ gho. $; 15=$ gdo $; 16=$ gal $; 17=$ gav $; 18=$ gag. 


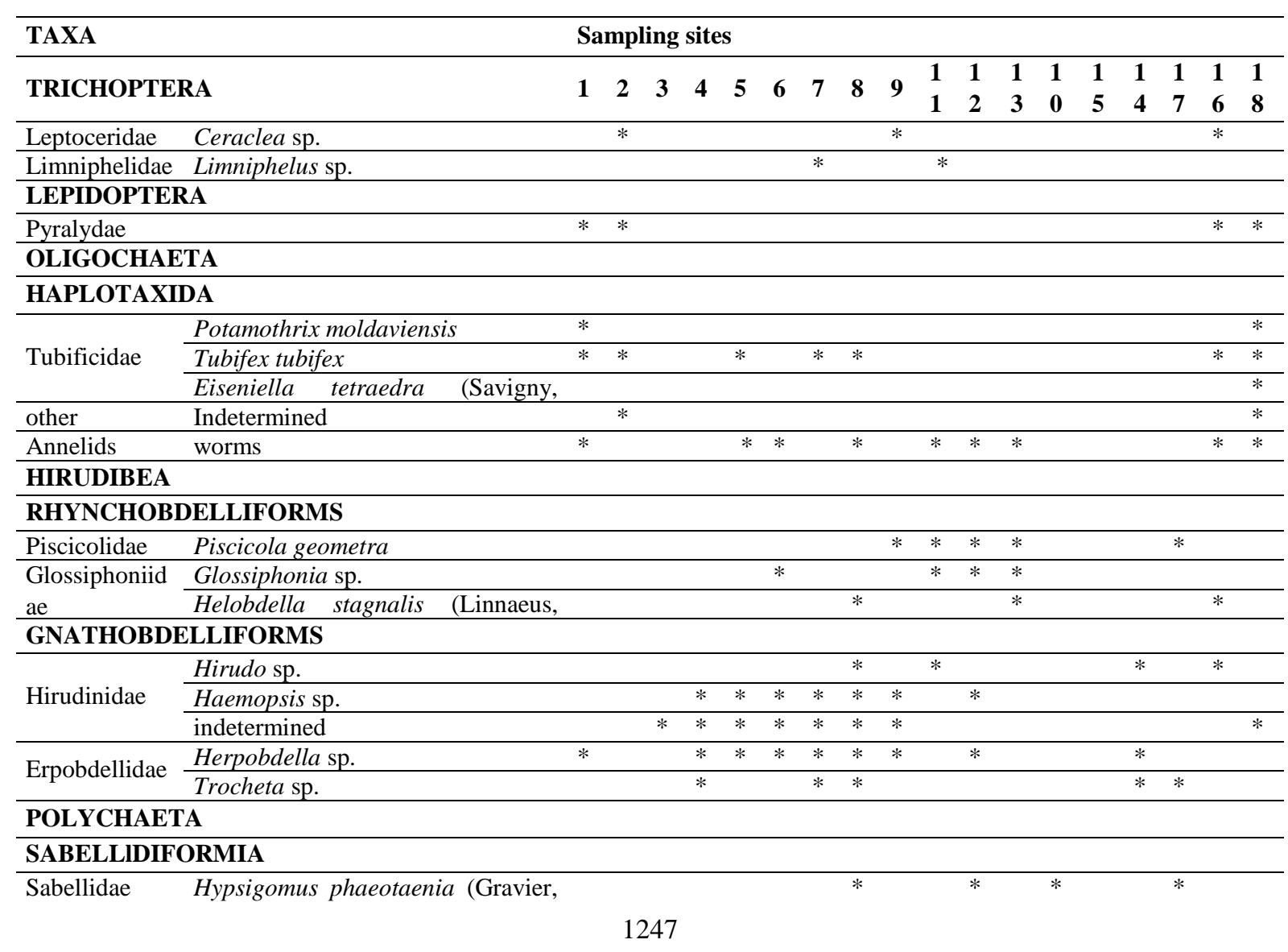


D. ADANDEDJAN et al. / Int. J. Biol. Chem. Sci. 6(3):1233-1252, 2012

\begin{tabular}{|c|c|c|c|c|c|c|c|c|c|c|c|c|c|c|c|c|c|c|c|}
\hline & 1908) & & & & & & & & & & & & & & & & & & \\
\hline & Sabella (Linne) sp. & & & & & & & & & & & * & & & & & & & \\
\hline \multirow{4}{*}{ Serpulidae } & $\begin{array}{l}\text { Ficopomatus enigmaticus (Fauvel, } \\
\text { 1923) }\end{array}$ & & & $*$ & & & & & * & * & & * & & * & & $*$ & * & * & \\
\hline & Ditrupa arietina (Müller, 1776) & & & & & & & & & & & & * & & * & & & & \\
\hline & Filograna sp. & & $*$ & & & & & & & & & & & & & & & & \\
\hline & Serpula (Linnaeus, 1767) sp. & * & $*$ & * & & * & * & * & $*$ & $*$ & & * & $*$ & * & & $*$ & $*$ & * & \\
\hline Pectinariidae & Pectinaria sp. & * & * & * & & & & & * & & & $*$ & & * & & & & * & \\
\hline \multirow[t]{2}{*}{ Naldanidae } & Clymene (Savigny, 1818) sp. & & & & & & & & & & & $*$ & & & & & & & \\
\hline & NEREIDIFORMIA & & & & & & & & & & & & & & & & & & \\
\hline \multirow{2}{*}{ Nephthtididae } & Nephthys (Cuvier, 1817) sp. & & $*$ & & & & * & & * & & & & & & & & * & * & \\
\hline & Eteone (Savigny, 1818) sp. & $*$ & $*$ & & & & & & & & & & & & & & & & \\
\hline \multirow{5}{*}{ Nereidae } & Nereis (Linne, 1758) sp. & $*$ & $*$ & $*$ & $*$ & & & $*$ & $*$ & $*$ & $*$ & $*$ & $*$ & $*$ & $*$ & $*$ & $*$ & $*$ & $*$ \\
\hline & Nereis diversicolor (Müller, 1776) & & $*$ & & & $*$ & $*$ & & $*$ & & & $*$ & $*$ & & & & & & \\
\hline & Nereis virens (Sars, 1835$)$ & & & & & & & & $*$ & & & * & & & & & & * & \\
\hline & Nereis pelagica (Fauvel, 1914) & & & & $*$ & & $*$ & $*$ & $*$ & & $*$ & & $*$ & $*$ & $*$ & * & $*$ & & \\
\hline & Perinereis (Kinber) sp. & & & & & & $*$ & & & & & & $*$ & & & & $*$ & $*$ & \\
\hline Glyceridae & Glycera (Savigny, 1818) sp. & & & & $*$ & $*$ & & & & & & & $*$ & $*$ & * & & & * & \\
\hline \multirow[t]{2}{*}{ Phyllodocidae } & Lopadorhynchus (Grube) sp. & & & & & & & & & & & $*$ & & & & & & & \\
\hline & Phyllodoce (Savigny, 1818) sp. & & & & & & & & $*$ & & & $*$ & $*$ & & & & & & \\
\hline Alciopidae & Callizona (Greeff, 1885) sp. & & & & $*$ & & * & & & & & & & & & * & & & \\
\hline Aréniolidae & Arenicola (Fauvel) sp. & & & & & $*$ & $*$ & $*$ & & & & & $*$ & $*$ & $*$ & & & & \\
\hline Ciratulidae & Dodecaeria (Oersted) sp. & & & & & & & & & & & $*$ & & & & & & & \\
\hline Syllidae & Syllis (Savigny, 1818) sp. & & & & & & & $*$ & & & & $*$ & $*$ & & & & & & \\
\hline \multicolumn{2}{|c|}{ ECHINODEIA } & & & $*$ & $*$ & & & & & & & & & & & & & & \\
\hline \multicolumn{2}{|l|}{ ARACHNIDS } & $*$ & $*$ & $*$ & & $*$ & $*$ & $*$ & & & & $*$ & & & & & & $*$ & $*$ \\
\hline \multicolumn{2}{|l|}{ Total } & 56 & 51 & 48 & 46 & 40 & 44 & 34 & 68 & 40 & 43 & 54 & 34 & 47 & 56 & 51 & 48 & 46 & 40 \\
\hline
\end{tabular}




\section{DISCUSSION}

The findings described above are consistent with similar information on biological communities of estuarine zone (Bazaïri et al., 2003). Taxonomic structure of macrobenthic organisms of the lagoon, is characterized by a common classical faunistic group of the estuaries and lagoons ecosystems. The lagoon is richer in Molluscs and Crustaceans represented by Tympanotonus fuscatus radula, Littorina africana, Neritina sp., Corbula trigona, Tagelus sp., Cassostrea gazar, Anadara senilis populations which mark the passage from marine ecosystems to coastal ecosystems of West-Africa (Le Loeuf, 1999). Numerous studies done in Africa (Bazaïri et al., 2003; Chaouti and Bayed, 2005; Kouadio et al., 2008) and elsewhere in the world (Marzano et al., 2003; Leonardo and Bemvenuti, 2006) in lagoons highlight the same results in transitional waters. This rich biodiversity and the ecological importance of estuarian ecosystems are favoured by their proximity with the sea that furnishes marine species through tidal inundations and mangroves trees (Zabi and Le Loeuf, 1993; Akin et al., 2003; Alfaro, 2006; Leonardo and Bemvenuti, 2006; Lee, 2008). Mangroves and seagrass habitats in estuarine systems provide more structures for potential invertebrates to settle on (Davis et al., 2001; Morrisey et al., 2002; Ellis et al., 2004; Alfaro, 2006).

The composition of the benthic macrofauna observed does not change along the lagoon. Several studies concerning the distribution of benthic macroinvertebrate such as those done by Jacobsen and Encalada (1998) in the Ecuadorian Lagoons, by Leonardo and Bemvenuti (2006) in the Patos Lagoon, Brasil, and by Sporkar et al. (2006) in the Stupavskypotok Lagoon, Central Europe, show the same results. In fact, Coimbra et al. (1996), Edia et al. (2007), Fishar and Williams (2008) showed that the benthic organisms of lagoons are particularly subjected to big and seasonal variations of the environmental parameters mostly to hydrological conditions. In the "Coastal Lagoon", the abundant freshwater inputs in the lagoon via the hydroelectrical factory in the upper zone and the different water discharges favour the proliferation of aquatic vegetation such as Eichhornia crassipes that covers the entire surface of water causing several problems to human activities (hampering navigation, obstruction fishing nets) and local anoxia (Marzano et al., 2003). It has been pointed out that aquatic vegetation favours the colonization of aquatic ecosystems by invertebrates such as Insects, Molluscs and Annelids (Jonathan et al., 2006). It is also demonstrated that aquatic vegetation determines a heterogeneous environment and provides shelter for invertebrates (Cogerino et al., 1995; Arab et al., 2004; Principe and Corigliano, 2006; Jonathan et al., 2006; Arimoro et al., 2007) and adequate feeding conditions since the probability of predation decreases (Lencioni and Rossaro 2005, Jonathan et al., 2006).

The macrobenthic fauna observed is also determined by a few predominant taxa. This result can be explained by the intensive and multiple anthropic activities of the people living along the lagoon. These activities are daily amplified due to population increase and to the difficult living conditions observed recently. Among the consequences is the reduction of the biodiversity marked by the important influx of organic matter which settles further downstream. The abundance of polluo-tolerant species such as Tympanotonus f. radula, Chironomus sp. demonstrates the important amount of organic matter in this lagoon. Also, the lack of numerous indicator taxa of a good water quality is a sign that the activities done along the lagoon have negative impacts on the biodiversity.

The upper side of the lagoon showed the highest diversity and abundance. This result is determined by the environmental conditions (sediment predominantly sandy, high concentration of DO). In fact, this part of the lagoon receives the water released from the hydroelectrical dam which mixes regularly 
with the lagoon water. This causes the salinity to decrease. The reduction of this parameter creates less stressful conditions to certain animals. These conditions are favourable to Insects life, freshwater Gastropoda and Bivalvia, the latter developing moderate concentration of salinity for its survival (Le Loeuf, 1999).

On the contrary, the lower part of the lagoon accumulates high quantities of organic matters loaded by different discharges (from rains and hydroelectrical factory) into the lagoon. The sediment from this sampling sites located near the mouth of the lagoon contains a major proportion of clay and silt because of the accumulation of organic enrichment contained in discharges. Actually, these sites are exposed to severe anthropic activities such as solid and liquid wastes, cultivation of oysters and molluscs that provide a supply of organic wastes to the lagoons. This enrichment in sediment with organic matter leads to the decrease of dissolved oxygen concentration (Rosenberg and Resh, 1993; Sornin, 1984) which favours the development of opportunistic species such as Ciratulidae and Tubificidae. This group of sites is near villages that receives intensive anthropic activities such as aquaculture and salt mining. The composition of the benthic fauna of this group reflects most of the one polluted zone where Gastropoda, Diptera, Hirudinea and Oligochaeta taxa are abundant.

Mature taxa such as Grapsidae, Portunidae, Gecarcinudae, Balanidae (Vacquier, 2007) dominate also the groups of sites near the mouth of the lagoon. These groups are discriminated by high values of salinity and its sediments are mostly muddy filled by shell wastes. Such environments are characterized by a low renewal of water. Temporary hypoxias are observed in these habitats and provoke the decrease of the macrofauna abundance and richness (Gray et al., 2002). In these sites, predations must be strong; only mature taxa can successfully deal with predators that could survive (Vacquier, 2007).

\section{Conclusion}

The information gathered during this study, the first one on the Macrobenthic organisms done in this lagoon, represents a valuable increase in our knowledge. Nevertheless, this baseline study only refers to spatial distribution of the benthic communities of the lagoon that is related mainly to changes in salinity and hydrology. These factors interact with other physical and chemical parameters on the distribution of the fauna observed. So, seasonal changes can occur, mainly driven by hydrological and geomorphological mechanisms. An increased river discharge in rainy seasons and decreased in dry seasons would lead to a different salinity structure of the lagoon. This in turn, would help understanding seasonal patterns of such a distribution. The study reveals mostly the ecological state of coastal lagoons and we assume that the benthic macroinvertebrates can be use as indicators. We also hope that these preliminary findings stimulate future research on the ecology of macroinvertebrate organisms, even in all aquatic ecosystems of the country and their use in the different conservation strategies.

\section{ACKNOWLEDGMENTS}

We extend our warm thanks to Dr D. Diomandé, Dr Y. Bony and their assistants in the Laboratory of Environment and Aquatic Biology (LEBA), University of AboboAdjamè, Abidjan, for taxonomic identifications of the macrofauna and advice; Dr E. O. Edia and Dr E. Konan of the Laboratory of Ecology and Applied Biology of the Abobo-Adjamè University (Ivory Coast) for statistical advice; Pr A. Ouattara for his intervention in reading and improving the first methodology for this project. Thanks to Pr. M. Oyede, ex Dean of Sciences and Technic Faculty (University of AbomeyCalavi) for advice and technical assistance. We also thank Dr C. Bonou for helping with sampling procedures in the field. Special thanks are due to the research group at the Laboratory of Hydrobiology and Aquaculture, 
Abomey-Calavi University, Benin who supported this project from the start. Special thanks to the reviewers for valuable suggestions of this manuscript.

This research was supported by funds from the interregional project ESTEL (Ecologie des Systèmes Tropicaux Estuariens et Lagunaires) at the University of AbomeyCalavi and the University of Abobo-Adjamè, in collaboration with the Polytechnic Institute of Toulouse, France, and financed by the French Africa University (AUF).

\section{REFERENCES}

Akin S, Winemiller KO, Gelwick FP. 2003. Seasonal and spatial variations in fish and macrocrustacean assemblage structure in Mad Island Marsh estuary, Texas. Estuar. Coast. Shelf Sci., 57: 269-282.

Alfaro AC. 2006. Benthic macroinvertebrate community composition within a mangrove/ seagrass estuary in Northern New Zealand. Estuar. Coast. Shelf Sci., 66: $97-110$.

Arab A, Lek S, Lounaci A, Park YS. 2004. Spatial and temporal patterns of benthic invertebrate communities in an intermittent river (North Africa). Ann. Limnol. - Int. J. Limnol., 40: 317-327.

Arimoro FO, Ikomi RB, Efemuna E. 2007. Macroinvertebrate community patterns and diversity in relation to water quality of river Ase, Níger Delta, Nigeria. $J$. Fish. Aquat. Sci. 2: 337-344.

Barbour MT, Gerritsen J, Griffith GE, Frydenborg R, McCarron E, White JS, Bastian ML. 1996. A framework for biological criteria for Florida streams using benthic macroinvertebrates. J. N. Am. Benthol. Soc. 15: 185-211.

Bazaîri H, Bayed A, Glémarec M, Hily C. 2003. Spatial organization of macrozoobenthic communities in response to environmental factors in a coastal lagoon of the NW African coast (Merja Zerga, Morocco). Oceanol. Acta, 26: 457-471.

Chalves ML, Rieradelvall M, Chainho P, Costa JL, Costa MJ, Prat N. 2008.
Macroinvertebrate communities of nonglacial high altitude intermittent streams. Fresh. Biol., 53: 55-76.

Chaouti A, Bayed A. 2005. Diversité taxonomique et structure de la macrofaune benthique des substrats meubles de la lagune de Smir [Taxonomic diversity and structure of the benthic macofauna od soft substrats of Smir Lagoon]. Trav. Inst. Sci., Rabat (Maroc) série générale $n^{\circ} 4: 33-42$.

Cogerino L, Cellot B, Bournaud M. 1995. Microhabitat diversity and associated macroinvertebrates in aquatic banks of a large European river. Hydrobiologia, 304: $103-115$.

Coimbra CN, Graqa MAS, Cortes RM. 1996. The effects of a basic effluent on macroinvertebrate community structure in a temporary Mediterranean river. Environ. Pollut., 94: 301-307.

Davis S, Childersn D, Day J, Rudnick D, Shlar F. 2001. Nutrients dynamics in vegetated and unvegetated areas of a southern Everglades mangrove creek. Estuar. Coast. Shelf Sci., 32, 1-2.

Edia OE, Brosse S, Ouattara A, Gourène G, Winterton P, Lek-Ang S. 2007. Aquatic insect assemblages patterns in four WestAfrican Coastal Rivers. J. Biol Sci., 7: 1130-1138.

Ellis J, Nicholls P, Craggs R, Hofstra D, Hewit J. 2004. Effects of terrigenous sedimentation on mangrove physiology and associated Macrobenthic communities. Mar. Ecol. Prog. Ser., 297: 71-82.

Fishar MR, Williams WP. 2008. The development of a Biotic Pollution Index for the River Nile in Egypt. Hydrobiologia, 598: 17-34.

Gray JS, Wu RSS, Or YY. 2002. Effects of hypoxia and organic enrichment on the coastal marine environment. Mar. Ecol. Prog. Ser., 238: 249-279.

Jacobsen and Encalada. 1998. The macroinvertebrates fauna of Ecuadorian high-land streams in the wet and dry seasons. Hydrobiol., 142: 53-70. 
Jonathan P, Hornung A, Foote L. 2006. Aquatic invertebrate responses to fish presence and vegetation complexity in western boreal wetlands, with implications for waterbird productivity. Wetlands., 26:1-12.

Kohonen T. 1982. Self-organized formation of topologically correct feature maps. Biol. Cybern., 43: 59-69.

Kohonen T. 1995. Self-Organizing Maps. Informatique Sciences, Series 30. Springer-Verlag: Heindelberg.

Kouadio KN, Diomandé D, Ouattara A, Koné YJM, Gourène G. 2008. Taxonomic diversity of benthic macroinvertebrates in Aby Lagoon (Ivory Coast, West Africa). Pak. J. Biol. Sci., 11(18): 2224-2230.

Lee SY. 2008. Mangroves macrobenthos: Assemblages, services and linkages. $J$. Sea Res., 59: 16-29.

Leonardo CR, Bemvenuti CE. 2006. Temporal variability of the estuarine macrofauna of the Patos Lagoon, Braz. Rev. Biol. Mar. Oceanogr., 41(1): 1-9.

Le Loeuf P. 1999. La macrofaune d'invertébrés benthiques des écosystèmes à salinité variable le long des côtes atlantiques de l'Afrique tropicale; variations de la biodiversité en relation avec les conditions climatiques actuelles (précipitations) et l'histoire climatique régionale. Zoosystema, 21(3): 557-571.

Lencioni V, Rossaro B. 2005. Microdistribution of chironomids (Diptera: Chironomidae) in Alpine streams: an autoecological perspective. Hydrobiologia, 533: 61-76.

Marzano CN, Liaci LS, Fianchini A, Gravina F, Mercurio M, Corriero G. 2003. Distribution, persistence and change in the macrobenthos of the lagoon of Lesina (Abulia, southern Adriatic Sea). Oceanol. Acta, 26: 57-66.

Morrisey DT, Skilleter GA, Ellis JI, Burns BR, Kemp CE, Burt K. 2002. Differences in benthic fauna and sediment among mangrove (Avicennia marina var australasica) stands of different ages in
New Zealand. Estuar. Coast. Shelf Sci., 56: 581-592.

Park YS, Céréghino R, Compin A, Lek S. 2003. Applications of artificial neural networks for patterning and predicting aquatic insects species richness in running waters. Ecol. Model., 160: 265-280.

Principe RE, Corigliano MC. 2006. Benthic, drifting and marginal macroinvertebrate assemblages in a lowland river: temporal and spatial variations and size structure. Hydrobiologia, 553: 303-317.

Rosa LCR, Bemvenuti CE. 2006. Temporal variability of the estuarine macrofauna of the Patos Lagoon, Braz Rev. Biol. Mar. Oceanogr., 41(1): 1-9.

Snelgrove PVR, Butman CA. 1994. Animalsediment relationships revisited: cause versus effect. Oceanogr. Mar. Biol. Annu. Rev., 32: 111-177.

Sporkar F, Hanneke V, Bulankova E, Krno I. 2006. Influence of seasonal variation on bioassessment of streams using macroinvertebrates. Hydrobiologia, 566: 543-555.

Sornin JM. 1984. Rôle et conséquences de la biodéposition à l'interface eau/sédiment. J. Rech. Oceanogr., 9: 38-40.

Vacquier C. 2007. Inventaire de la macrofaune benthique du golfe de Beauduc en vue de l'élaboration d'un protocole de suivi biologique du littoral marin du parc naturel, régional de Camargue. Master Professionnel Marseille. Université de la Méditerranée, Marseille, 65 p.

Vesanto J, Himberg J, Alhoniemi E, Parhankangas J. 1999. Self-organizing map in Matlab: the SOM Toolbox. Proceedings of the Matlab DSP Conference Espoo: Finland, Comsol Oy; 35-40.

Zabi GS, Le Loeuf P. 1993. Revue des connaissances sur la faune benthique des milieux margino-littoraux d'Afrique de l'ouest. Deuxième partie: peuplements et biotopes Rev. Hydrobiol. Trop., 26(1): 19-51. 